\title{
Project 1017633
}

\section{New Catalytic DNA Biosensors for Radionuclides and Metal ions}

\author{
Lu, Yi \\ University of Illinois
}

\begin{abstract}
RESULTS TO DATE: Since the last report, we have made the following progresses in the project.
\end{abstract}
1. In vitro selection for DNAzymes that are specific for uranium.

In vitro selection for DNAzymes that are catalytically active with UO22+ ions as the metal cofactor has been completed. The 10th generation pool of DNA was cloned and sequenced. A total of 84 clones were sequenced and placed into families based on sequence alignments. Selected members of each family were 5-labeled with 32P and amplified using PCR. Activity assays were conducted using the isotopically labeled DNAzymes in order to determine which sequences were the most active. The secondary structures of the two most active sequences, called Clone 13 and Clone 39, were determined using the computer program Mfold. A cleavage rate of approximately $1 \mathrm{~min}-1$ in the presence of 10 uM UO22+ was observed for both clones. Clone 39 was determined to be the best candidate for truncation to create a trans-cleaving DNAzyme, based on its secondary structure. An enzyme strand, called 39E, and a substrate strand, called 39DS, were designed by truncating the cis-cleaving DNAzyme. An alternative enzyme strand, called 39Ec, was also assayed with the 39DS substrate. This strand was designed so that the two binding arms were perfectly complimentary, unlike 39E, which formed three mismatched base pairs with 39DS. Both 39E and 39Ec were found to be active, with a rate of approximately 1 min-1 in the presence of 10 uM UO22+. A preliminary UO22+ binding curve was obtained for the 39Ec/39DS trans-cleaving system. The enzyme is active with UO22+ concentrations as low as $1 \mathrm{nM}$. Based on the preliminary binding curve data, the apparent UO22+ binding constant is approximately $330 \mathrm{nM}$, and $\mathrm{kmax}$ is approximately $1 \mathrm{~min}-1$.

\section{Detailed Characterization of the DNAzyme/nanoparticle System towards Optimized Detection} Conditions.

We previously communicated a method for directed assembly of gold nanoparticles using a Pb2+dependent DNAzyme and demonstrated the application of this system as a colorimetric biosensor. The sensor shows high sensitivity and selectivity toward $\mathrm{Pb} 2+$, and undergoes a blue-to-red color transition in the presence of $\mathrm{Pb} 2+$. To gain a deeper insight into the analyte-directed nanomaterials assembly and sensing processes, a detailed characterization of the system has been performed. First, we found that the presence of gold nanoparticles had no effect on the Pb2+-dependent activity of the DNAzyme and the presence of DNAzyme has little effect on the melting properties of the DNA-functionalized nanoparticle aggregates, suggesting that the performance of the nanoparticle and DNAzyme systems can be optimized independently. Second, the optimal length of the DNAzyme and the alignment of the DNAfunctionalized gold nanoparticles for the assembly and sensing processes have been determined to be 9 base pairs on each end for the DNAzyme, and Head-to-Tail alignment for the DNA-functionalized gold nanoparticles. Third, the optimal stoichiometry of the enzyme to the substrate strands of the DNAzyme was shown to be one to one in nanoparticle aggregates. Finally, the most favorable temperature and $\mathrm{pH}$ conditions for the system have also been established, with a temperature of $37 \mathrm{C}$ and $\mathrm{pH}$ of 6.4 to 9.2 as the best operating conditions. The study also revealed that, for most efficient assembly of nanoparticles, the DNA backbone should be rigidified by formation of double helix with other DNA molecules. These findings allow optimization of the processes for directed assembly of nanomaterials and for colorimetric sensing. The results lead to a better understanding of the system for further improvement of the sensor and a paper describing the results is published (Chem. Mater. 16, 3231-3238 (2004)).

3. Improving the colorimetric $\mathrm{Pb} 2+$ sensor by changing the alignment and size of nanoparticles. 
The combination of high metal selectivity of DNAzymes with the strong distance-dependent optical properties of metallic nanoparticles has presented considerable opportunities for designing colorimetric sensors for metal ions. We previously communicated a design for a colorimetric lead sensor based on the assembly of gold nanoparticles by a Pb2+-dependent DNAzyme. However, heating to $50 \mathrm{C}$ followed by a cooling process of $\sim 2$ hours was required to observe the color change. Herein we report a new improved design that allows fast (<10 minutes) detection of $\mathrm{Pb} 2+$ at ambient temperature. This improvement of sensor performance is a result of detailed studies of the DNAzyme and nanoparticles, which identified tail-to-tail nanoparticle alignment, and large ( $42 \mathrm{~nm}$ diameter) nanoparticle size as the major determining factors in allowing fast color changes. The optimal conditions for other factors such as temperature (35 C) and concentrations of the DNAzyme (2 uM), its substrate $(3 \mathrm{nM})$, and $\mathrm{NaCl}(300 \mathrm{mM})$ have also been determined. These results demonstrate that fundamental understanding of the DNAzyme biochemistry and nanoparticle science can lead to dramatically improved colorimetric sensors. With these changes, we achieved fast detection (less than 10 minutes) at ambient temperatures. The results were published in J. Am. Chem. Soc. 126, 12298-12305 (2004). 4. Immobilization of a Catalytic DNA Molecular Beacon on $\mathrm{Au}$ for $\mathrm{Pb}(\mathrm{II})$ Detection.

$\mathrm{A} \mathrm{Pb}(\mathrm{II})$-specific DNAzyme fluorescent sensor has been modified with a thiol moiety in order to immobilize it on a Au surface. Self-assembly of the DNAzyme is accomplished by first adsorbing the single thiolatedenzyme strand (HS-17E-Dy) followed by adsorption of mercaptohexanol (MCH), which serves to displace any Au-N interactions and ensure that DNA is bound only through the S-headgroup. The pre-formed selfassembled monolayer (SAM) is then hybridized with the complementary fluorophore-containing substrate strand (17DS-FI). Upon reaction with $\mathrm{Pb}(\mathrm{II})$, the substrate strand is cleaved, releasing a fluorescent fragment for detection. Fluorescence intensity may be correlated with original $\mathrm{Pb}$ (II) concentration, and a linear calibration was obtained over nearly four decades: from $10 \mathrm{uM}$ to $1 \mathrm{nM}$. The immobilized DNAzyme is a robust system; it may be regenerated after cleavage, allowing multiple sensing cycles. In addition, drying of fully assembled DNAzyme before reaction with $\mathrm{Pb}$ (II) does not significantly affect analytical performance. These results demonstrate that, in comparison with solution-based schemes, immobilization of the DNAzyme sensor onto a Au surface lowers the detection limit (from $10 \mathrm{nM}$ to $1 \mathrm{nM}$ ), maintains activity and specificity, allows sensor regeneration and long-term storage. Realization of $\mathrm{Pb}$ (II) detection through an immobilized DNAzyme is the first important step toward creation of a stand-alone, portable $\mathrm{Pb}$ (II) detection device such as those immobilizing DNAzyme recognition motifs in the nanofluidic pores of a microfluidic-nanofluidic hybrid multilayer device. These results were published in Anal. Chem. 77, 442448 (2005).

DELIVERABLES: 1. Juewen Liu and Yi Lu, Optimization of a Pb2+-Directed Gold Nanoparticle/DNAzyme Assembly and Its Application as a Colorimetric Biosensor for Pb2+, Chem. Mater. $16,3231-3238$ (2004)

2. Juewen Liu and Yi Lu, Accelerated Color Change of Gold Nanoparticles Assembled by DNAzymes for Simple and Fast Colorimetric Pb2+ Detection, J. Am. Chem. Soc. 126, 12298-12305 (2004)

3. Carla B. Swearingen, Daryl P. Wernette, Donald M. Cropek, Yi Lu, Jonathan V. Sweedler, and Paul W. Bohn, Immobilization of a Catalytic DNA Molecular Beacon on Au for Pb(II) Detection, Anal. Chem. 77, 442-448 (2005).

4. Juewen Liu and Yi Lu, Detecting Metal Ions with Fluorescent DNAzyme Biosensors, Methods in Molecular Biology (in press).

5. Juewen Liu and Yi Lu, Multi-Fluorophore FRET for Probing Nucleic Acids Folding and Structure, Methods in Molecular Biology (in press). 